\title{
The study of threshold determination of gene identification and its improvement algorithms
}

\author{
Chun Yan ${ }^{\mathrm{a}}$, Wei Liu ${ }^{\mathrm{b}, *}$, Yiwei Qiu ${ }^{\mathrm{a}}$ and Haitang Sun ${ }^{\mathrm{a}}$ \\ ${ }^{a}$ College of Mathematics and System Sciences, Shandong University of Science and Technology, \\ Qingdao Shandong 266590, China \\ ${ }^{\mathrm{b}}$ College of Information Science and Engineering, Shandong University of Science and Technology, \\ Qingdao Shandong 266590, China
}

\begin{abstract}
The problem of gene recognition based on the ratio of power spectrum, SNR, and Gabor transform and its implementation of the calculation were discussed. The optimal threshold could guarantee to identify the DNA sequences with the signal-to-noise ratio. It summarized three kinds of traditional ways to determine the threshold, and advanced the optimum entitled method showing the disparate degrees of highlight and the discrimination rate method of the exons or introns as far as possible to improve the rate of their accuracy. To evaluate different determination methods of threshold by using the calculation results of four kinds of DNA sequence. In order to ensure the analysis of DNA sequence more accurate, it adopted and improved gene identification method of Fourier transformation in a short time which is based on Gabor transformation. By using of the ergodic theory, the fixed percentage of the sequence length of exons in DNA has been improved to be the dynamic percentages which focus on different gene types. The exons of the DNA sequence which have been already discovered were identified by using the improved algorithm. With comparison of the results and the actual endpoint of exons, it confirmed that the improved algorithm can figure out the endpoint of the exons more accurate.
\end{abstract}

Keywords: The discrete Fourier transform, Gabor transform, gene identification, signal-to-noise ratio, threshold

\section{Introduction}

DNA is the carrier of biological genetic information. It uses genetic code to store information, and guides the synthesis of proteins. The accurate deliver of genetic information of protein could make the various life functions completely. Along with the successful completion of world human genome project, getting rich biological information from large amounts of DNA sequences through physical or mathematical methods has important theoretical significance and practical value in biology, medicine, pharmacy etc., and it is currently a hot research topic in the field of information biology. To identify the coding sequence of a given DNA sequence that namely exons, which is also called the gene prediction, is one of the most basic and important not solved completely problem in the field of

\footnotetext{
*Address for correspondence: Wei Liu, College of Information Science and Engineering, Shandong University of Science and Technology, Qingdao 266590, China, Tel.: +86 15063992767; Fax: +86 0532 86057638; E-mail: liuwei_doctor@yeah.net.
} 
information biology.

Firstly, this essay will discuss the threshold determination of different species types of genes. To determine the threshold of genes types in different species, and to study the threshold determination method of each kind of representative gene sequence exons, and determine the threshold. And through the exploring the classification effectiveness of exons and non-exons, we can make an analysis on classified error in the process of classification recognition.

For DNA sequences with specific gene types, it has some experience and subjectivity to set the discriminant threshold of SNR R as $R_{0}=2$. The discriminant threshold we chose should according to different gene types. The traditional determination method of threshold includes weight method and optimization method [1-5]. But considering the different needs of exons and introns, there isn't a reasonable solution. This essay summarized the existing methods, and found a more reasonable and accurate method of threshold determination method to enhance the accuracy in gene recognition.

Secondly, this essay will study the realization of the gene identification algorithm. Our purpose is to detect and report all the genes coding sequences in DNA sequence which have not yet been annotated and complete (exons). There are usually two kinds of gene identification algorithms using SNR, one is the moving window method with fixed length [6,7], and another is the recognition method of mobile signal-to-noise ratio curve [8-13]. At present, the gene recognition algorithms above can't get rid of the influence of random noise of DNA sequence, so it is difficult to determine the two endpoints of gene exons interval accurately.

We can consider the window method of the signal processing, use the Gaussian window function to deal with DNA indicated sequence, and determine the optimal threshold, thus determine the two endpoints of exons interval. And then to realize the gene identification algorithm, design and evaluate the accuracy of gene recognition algorithm, and further to forecast and analyze the six DNA sequence exonic range which are not annotated in gene6.

\section{The illustration of model assumptions and symbols}

\subsection{Model assumptions}

(1) The length of DNA sequences to be identified is a multiple of three, and intercept a fragment of length $k_{t}$ ( $k_{t}$ is a multiple of 3 ), and deem $k_{t}$ has a 3-cyclical. (2) No gene mutation and substitution exist. (3) The pulse signal of DNA sequence indicated is not stationary. (4) There are no influences among the bases far from each other.

\subsection{Symbolic description}

Let A denotes Adenine, $\mathrm{G}$ denotes Guanine, $\mathrm{C}$ denotes Cytosine, $\mathrm{T}$ denotes Thymine, I denotes the collection of four nucleotides, $\{S[n]\}$ denotes DNA sequence, $\left\{u_{b}[n]\right\}$ denotes indicate sequence of nucleotide $\mathrm{B},\left\{u_{b}[K]\right\}$ denotes the Fourier transform of indicate sequence of nucleotide $\mathrm{B},\{P[k]\}$ denotes power spectral sequence of DNA sequence, $\bar{E}$ denotes Average of power spectrum, L denotes DNA sequence length, $\mathrm{M}$ denotes the coefficient matrix of quadratic form, $p_{i}$ denotes length of DNA fragment, $\mathrm{V}$ denotes the two order central matrix. 
Table 1

Existing ways to determine threshold

\begin{tabular}{|c|c|c|c|c|}
\hline $\begin{array}{l}\text { Experience } \\
\text { method }\end{array}$ & $R_{\mathrm{o}}=2$ & & & \\
\hline \multirow[t]{10}{*}{ weight method } & weight & & \multirow[t]{2}{*}{ Threshold value $R_{0}$} & \multirow[t]{2}{*}{ Remarks } \\
\hline & $w_{1}$ & $w_{2}$ & & \\
\hline & 1 & 1 & \multirow{2}{*}{$R_{0}=\frac{E_{1}+E_{2}}{2}$} & \multirow[t]{2}{*}{-} \\
\hline & $\overline{2}$ & $\overline{2}$ & & \\
\hline & $E_{1}$ & $E_{2}$ & \multirow{2}{*}{$R_{0}=\frac{E_{1}}{E_{1}+E_{2}} E_{1}+\frac{E_{2}}{E_{1}+E_{2}} E_{2}$} & \multirow{2}{*}{$\begin{array}{l}\text { Mean value of exon } E_{1} \\
\text { Mean value of intron } E_{2}\end{array}$} \\
\hline & $\overline{E_{1}+E_{2}}$ & $E_{1}+E_{2}$ & & \\
\hline & $\sigma_{1}$ & $\sigma_{2}$ & \multirow{2}{*}{$R_{0}=\frac{\sigma_{1}}{\sigma_{1}+\sigma_{2}} E_{1}+\frac{\sigma_{2}}{\sigma_{1}+\sigma_{2}}$} & \multirow{2}{*}{$\begin{array}{l}\text { SD of exon } \sigma_{1} \\
\text { SD of intron } \sigma_{2}\end{array}$} \\
\hline & $\sigma_{1}+\sigma_{2}$ & $\sigma_{1}+\sigma_{2}$ & & \\
\hline & $m_{1}$ & $m_{2}$ & \multirow{2}{*}{$R_{0}=\frac{m_{1}}{m_{1}+m_{2}} E_{1}+\frac{m_{2}}{m_{1}+m_{2}} E_{2}$} & \multirow{2}{*}{$\begin{array}{l}\text { Median of exon } m_{1} \\
\text { Median of intron } m_{2}\end{array}$} \\
\hline & $m_{1}+m_{2}$ & $m_{1}+m_{2}$ & & \\
\hline $\begin{array}{l}\text { Optimizati-on } \\
\text { method }\end{array}$ & \multicolumn{3}{|c|}{$\max f(x)=\sum_{i} \operatorname{sgn}\left(R_{i}^{1}-R_{0}\right)+\sum_{j} \operatorname{sgn}\left(R_{0}-R_{j}^{2}\right)$} & $\begin{array}{l}R_{i}^{1} \in S_{1}, S_{1} \text { is the } \mathrm{SNR} \\
\text { aggregation of exon; } \\
R_{j}^{1} \in S_{2}, S_{2} \text { is the } \mathrm{SNR} \\
\text { aggregation of intron. }\end{array}$ \\
\hline
\end{tabular}

\section{Threshold determination of different species types of gene}

\subsection{Traditional ways to determine the thresholds}

Major ways to determine threshold can be summarized from references, such as experience method, weight method and optimization method. They are shown in Table 1.

The methods above are equivalent to estimate the exons and introns. In the optimization method, if the value of $R_{0}$ is small enough, exons can be misjudged as introns and the accuracy may be decreased, although the exons contained are as many as possible. In the similar way, if the value of $R_{0}$ is big enough, the misjudgment also happens frequently. If we need exons or introns controlled in the range which thresholds determined as many as possible, or the accuracy can be increased as much as possible, the results of the methods above may be less-than-desirable. So we give the improvement method as follows.

\subsection{Modified ways to determine the threshold}

\subsubsection{Weighting optimization method}

The aim of this method is to make exons or introns be contained in the sequence that have determined before as many as possible. The calculation equation is:

$$
\max f(x)=w_{1} \sum_{i} \operatorname{sgn}\left(R_{i}^{1}-R_{0}\right)+w_{2} \sum_{j} \operatorname{sgn}\left(R_{0}-R_{j}^{2}\right)
$$


In this equation, $w_{1} / w_{2}$ means the importance of exons or introns. The smaller of $w_{1} / w_{2}$, the more important exons are; the bigger of $w_{1} / w_{2}$, the more important introns are.

\subsubsection{Discriminant rate method of exons and introns}

The aim of this method is to make the accurate rate of exons or introns in the discriminant result reach the highest level.

$$
g_{1}(x)=\max \frac{\sum_{i} \operatorname{sgn}\left(R_{i}^{1}-R_{0}\right)}{\sum_{i} \operatorname{sgn}\left(R_{i}^{1}-R_{0}\right)+\sum_{j} \operatorname{sgn}\left(R_{j}^{2}-R_{0}\right)}, g_{2}(x)=\max \frac{\sum_{j} \operatorname{sgn}\left(R_{j}^{2}-R_{0}\right)}{\sum_{i} \operatorname{sgn}\left(R_{i}^{1}-R_{0}\right)+\sum_{j} \operatorname{sgn}\left(R_{j}^{2}-R_{0}\right)}
$$

If the accurate rate of exons needs to be high, equation $g_{1}(x)$ will be used. In the contrary, equation $g_{2}(x)$ will be used. The choice of different rate determined methods is based on the aim of the discrimination.

\subsection{Evaluation index of threshold discriminant effect}

There are three kinds of methods for determining the threshold above, but which kind of method is the best? Now suppose that the SNR classify threshold is $R_{0}$, that is to say $R \geq R_{0}$ is used as the discriminant rule for exons and $R \geq R_{0}$ is the discriminant rule for introns. Three kinds of index for evaluating the method of threshold discriminant, which were based on different types and different objective, were presented as follows.

(1) Discriminant rate of exons (or introns)

Most of the time, it is hoped that exons (or introns) in the DNA sequence can be determined as much as possible, even sometime introns are wrongly discriminated as exons or exons are wrongly discriminated as introns .

Discriminant rate of exons is $S_{n}=T_{P} /\left(T_{P}+F_{N}\right)$. Discriminant rate of introns is $S_{p}=T_{N} /\left(T_{N}+F_{P}\right)$. In the equation, $T_{P}$ represents the number of exons that are discriminated correctly; $T_{N}$ represents the number of introns that are discriminated correctly. $F_{N}$ is the number of exons that are discriminated as introns by error, and $F_{P}$ is the number of introns that are discriminated as exons by error.

(2) Total correct rate $A_{c}=\frac{S_{n}+S_{p}}{2}$

(3) The accuracy of exons in the discriminant result

Sometimes the total accuracy is not hoped to be large. In the contrary, the real content of exons in the discriminated ones is more valued. So the equation of accuracy of exons is defined as follows:

$$
B_{c}=\frac{T_{P}}{\left(T_{P}+F_{P}\right)}
$$

In this equation, the meanings of $T_{P}$ and $F_{P}$ are as same as above. 
Table 2

Comparison chart of determining threshold methods

\begin{tabular}{|c|c|c|c|c|c|}
\hline genes & & Human beings & Yeast of liquor & $\begin{array}{l}\text { Nematode's } \\
\text { cosmidT24C4 }\end{array}$ & Arabidopsis \\
\hline \multirow[t]{4}{*}{ Weight method } & $R_{0}^{1}$ & 1.6156 & 3.9612 & 2.1781 & 1.3421 \\
\hline & $A_{c}$ & 0.7318 & 0.7858 & 0.7523 & 0.7011 \\
\hline & $B_{c}$ & 0.7657 & 0.7978 & 0.7352 & 0.7312 \\
\hline & $S_{n}$ & 0.7836 & 0.7931 & 0.7452 & 0.6892 \\
\hline \multirow{4}{*}{$\begin{array}{l}\text { Weighted average } \\
\text { method with SD }\end{array}$} & $R_{0}^{1}$ & 2.295 & 6.5213 & 4.2518 & 1.7231 \\
\hline & $A_{c}$ & 0.6601 & 0.7018 & 0.6813 & 0.6621 \\
\hline & $B_{c}$ & 0.8073 & 0.8241 & 0.7693 & 0.7511 \\
\hline & $S_{n}$ & 0.7981 & 0.8013 & 0.7658 & 0.7541 \\
\hline \multirow[t]{4}{*}{ Optimization method } & $R_{0}^{1}$ & 1.1201 & 1.5621 & 1.1476 & 1.1135 \\
\hline & $A_{c}$ & 0.7823 & 0.9011 & 0.8312 & 0.7745 \\
\hline & $B_{c}$ & 0.8231 & 0.8567 & 0.8015 & 0.7553 \\
\hline & $S_{n}$ & 0.7731 & 0.7818 & 0.7743 & 0.7217 \\
\hline \multirow{4}{*}{$\begin{array}{l}\text { Weighted } \\
\text { optimization method } \\
\text { with SD }\end{array}$} & $R_{0}^{1}$ & 1.8762 & 1.8931 & 2.0176 & 1.2431 \\
\hline & $A_{c}$ & 0.7412 & 0.8123 & 0.7564 & 0.7634 \\
\hline & $B_{c}$ & 0.8169 & 0.8419 & 0.7884 & 0.7451 \\
\hline & $S_{n}$ & 0.7681 & 0.7519 & 0.8031 & 0.7018 \\
\hline \multirow{4}{*}{$\begin{array}{l}\text { Discriminant rate } \\
\text { method of exons }\end{array}$} & $R_{0}^{1}$ & 0.8962 & 1.1213 & 1.1108 & 0.9852 \\
\hline & $A_{c}$ & 0.6891 & 0.7421 & 0.6985 & 0.7125 \\
\hline & $B_{c}$ & 0.8921 & 0.9314 & 0.8216 & 0.7947 \\
\hline & $S_{n}$ & 0.9012 & 0.9218 & 0.8321 & 0.8016 \\
\hline \multirow{4}{*}{$\begin{array}{l}\text { SNR threshold value } \\
\text { is fixed as } 2\end{array}$} & $R_{0}^{1}$ & 2 & 2 & 2 & 2 \\
\hline & $A_{c}$ & 0.6768 & 0.8898 & 0.7269 & 0.6572 \\
\hline & $B_{c}$ & 0.6731 & 0.7076 & 0.653 & 0.6012 \\
\hline & $S_{n}$ & 0.6834 & 0.7215 & 0.7018 & 0.6547 \\
\hline
\end{tabular}

\subsection{Analysis and assess of the methods used for determining threshold}

In order to assess the methods of determining threshold and compare them with the method of using a fixed threshold value about 2, we chose 4 representative groups of gene sequences based on the famous biological data sites: http://www.ncbi.nlm.nih.gov/guide/. The accuracy statistics can be shown in Table 2.

By analysis the date of Table 2, some can be discovered: Accuracy of weight method, weighted average method with SD and threshold value is fixed as 2 is less than the accuracy of optimization method. The percentage of real exons in discriminant rate method of exons is larger than other methods. Weighted optimization method with SD is the best to find introns when the introns are regarded as important. The threshold value in discrimination rate method of exons is the smallest one.

\section{Improving of the gene recognition method}

\subsection{Disadvantages of the traditional method}




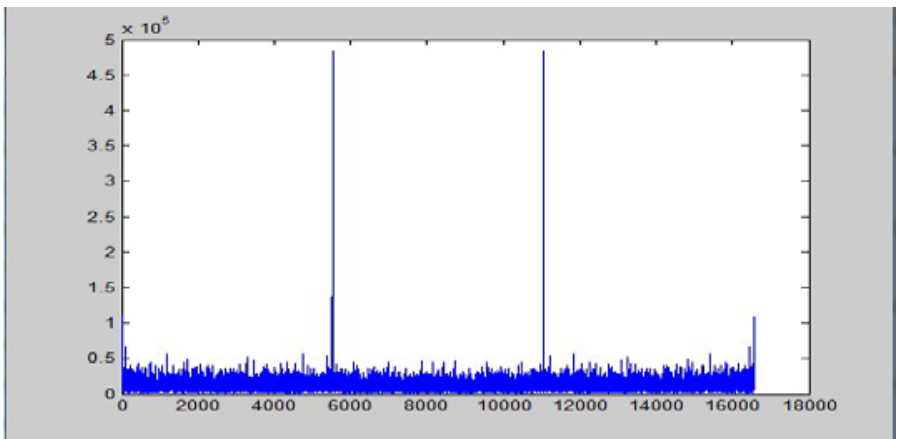

Fig. 1. The human mitochondrial genome sequence of DNA power spectrum.

Table 3

The table of exon actual position

\begin{tabular}{llllll}
\hline Serial number & Exon position & Serial number & Exon position & Serial number & Exon position \\
\hline 1 & $3307-4262$ & 6 & $8527-9207$ & 11 & $12337-14148$ \\
2 & $4470-5511$ & 7 & $9207-9990$ & 12 & $14149-14673$ \\
3 & $5904-7445$ & 8 & $10059-10404$ & 13 & $14747-15887$ \\
4 & $7586-8269$ & 9 & $10470-10766$ & & \\
5 & $8366-8572$ & 10 & $10760-12137$ & & \\
\hline
\end{tabular}

The traditional Fourier transform method has a high time complexity, and because of the influence of DNA random series and so on, it's difficult to find the two endpoints of exon interval accurately. The power spectra of human mitochondrial genome DNA sequence (length is 16569bp) using the traditional Fourier transform method is shown in Figure 1.

The actual position of Exon in human mitochondrial genome DNA sequence can be seen in Table 3 .

According to the Figure 1 and Table 3, we can obviously see that crest appears at the position of exons in this DNA sequence. But there is too much noise in its background, we cannot accurately figure out the exact endpoint on both sides of the crest. And it correctly identified only 2 of them in 13 exons. The correct rate is too low.

\subsection{Improving of the gene recognition method}

The short-time Fourier transform gene recognition method based on Gabor transform is given as follows. And it shows that the influence of background noise can be greatly reduced and it has a great accuracy through the data and figures.

\subsubsection{Weighting optimization method}

(1) Time-domain index

The average time in analysis window is $\bar{t}=1 /\|w(t)\|^{2} \int_{\infty}^{\infty} t|w(t)|^{2} d t$, which represents the "center of gravity" position of signal energy respected to time.

The duration of signal or the equivalent time width satisfies $(\Delta t)^{2}=\sigma_{t}^{2}=1 /\|w(t)\|^{2} \int_{-\infty}^{\infty}(t-\bar{t})^{2}|w(t)|^{2} d t$. if $\Delta t$ is small, it means that the signal energy is concentrated around the average time. With the increasing time, signal energy will definitely decay at a higher speed, and the analysis window is a short-duration signal which has a better temporal resolution. On the contrast, if $\Delta t$ is large, it means 
the analysis window is a long duration signal, which has a poor temporal resolution.

(2) Localized index in frequency domain

The average frequency of the analysis window is $\bar{\omega}=1 /\|\hat{w}(t)\| \int_{-\infty}^{\infty} \omega|\hat{w}(\omega)|^{2} d \omega$, and $\hat{w}(\omega)$ is the continuous Fourier transform of the analysis window $w(t)$. Continuous width of the frequency domain or the signal bandwidth satisfies $(\Delta \omega)^{2}=\int_{-\infty}^{\infty}(\omega-\bar{\omega})|\hat{w}(\omega)|^{2} d \omega$.

(3) Short-time Fourier transform

Using a window function on the signal to be measured, we analyzed the signal intercepted by the window once the window was panned, and this process is called short-time Fourier transform. This method can not only get the information about the frequency domain of the signal to be measured, but also observe the information of corresponding time domain. And because the shape of window remains unchanged during the transform process, it won't change the resolution of the time-frequency of the signal to be measured.

(4) Uncertainty principle

The limit of uncertainty principle is $\Delta x \cdot \Delta \omega \geq 1 / 2$, and the necessary and sufficient conditions for holding the quality is $W(x)=c e^{j \beta x} e^{-x^{2} / 4 a}$. Any window function of short-time Fourier transform will be subject to restrictions on uncertain principle.

(5) Gabor transform

The transform of using short-time Fourier transform with Gaussian-type window function is called Gabor transform. The Gaussian function is $g_{a}(x)=1 / 2 \sqrt{\pi a} e^{-x^{2} / 4 a}$. When $a>0$, the expression of the time window and frequency window are:

$$
\left\{\begin{array}{c}
t^{*}=\frac{1}{\|g\|_{2}^{2}} \int_{R} x|g(x)|^{2} d x \\
\bar{\omega}^{2}=\frac{1}{\|g\|_{2}^{2}} \int_{R} \bar{\omega}|g(\bar{\omega})|^{2} d \bar{\omega}
\end{array}\right\} .
$$

\subsubsection{Improving of the gene identification algorithm}

(1) Determine the peak point

(1)Determine the center of time window and frequency window of Gaussian-type window function.

(2) For gene sequence $\{S\}$, in where $a_{i}$ can be any value of parameter $a$, using the method of shorttime Fourier transform can get a new sequence $\left\{S_{i}\right\}$.

(3) Superimposing all the new sequence $\left\{S_{i}^{\prime}\right\}$ obtained from all parameter $a_{i}$ to get a new sequence $\left\{S^{\prime}\right\}$. The peak points of sequence $\left\{S^{\prime}\right\}$ are the exons of DNA fragment.

(2) Determine the endpoint of exons

(1) Get the sequence $\{R N S\}$ by sequencing the detected sequence $\{R N\}$ of DNA fragment in ascending order.

(2) Determine the threshold value. Use the corresponding element of $s s(s s=c \times n+1)$ in the sequence $\{R N S\}$ as the threshold. And assign the elements less than the threshold in sequence $\{R N\}$ as 0 . 
(3) The nonzero positions in $\{R N\}$ are the positions of exons. The zero positions are connection endpoints of exons.

(3) Improving of the model algorithm

The general value of $\mathrm{c}$ in $s s(s s=c \times n+1)$ is 0.85 , then we will improve it. Define $c_{i} \in(0,1)$, and there is only one threshold $R_{i 0}$ corresponded for $\forall c_{i}$.

\subsubsection{Evaluation on improved gene identification algorithm}

Use the improved algorithm to deal with the DNA sequence of human mitochondrial genome, and evaluate the algorithm with calculated results.

(1) Peak position.

A graph of the corresponding results for each base position is shown in Figure 2. The peak position is the position of exons.

(2) Fix the value of $c_{i}$

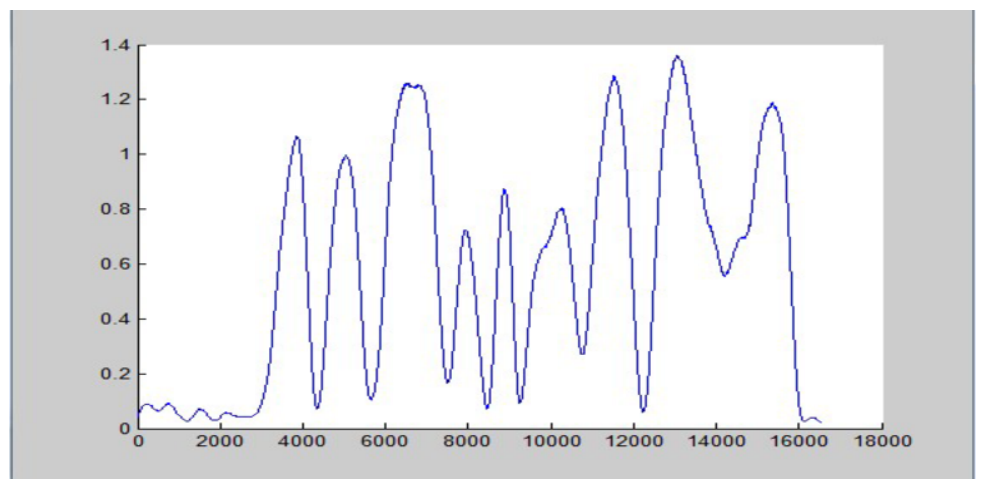

Fig. 2. The human mitochondrial genome sequence of DNA Gabor transformation curve.

Table 4

The table of human mitochondrial genome DNA sequence value of decision

\begin{tabular}{|c|c|c|c|}
\hline Value i & value $c_{i}$ & $\begin{array}{l}\text { Number of successful } \\
\text { predicted exons } n_{i}\end{array}$ & accuracy $p_{i}$ \\
\hline 1 & 0.30 & 5 & $38.46 \%$ \\
\hline 2 & 0.35 & 5 & $38.46 \%$ \\
\hline 3 & 0.40 & 6 & $46.15 \%$ \\
\hline 4 & 0.45 & 6 & $46.15 \%$ \\
\hline 5 & 0.50 & 7 & $53.85 \%$ \\
\hline 6 & 0.55 & 9 & $69.23 \%$ \\
\hline 7 & 0.60 & 8 & $61.54 \%$ \\
\hline 8 & 0.65 & 7 & $53.85 \%$ \\
\hline 9 & 0.70 & 6 & $46.15 \%$ \\
\hline 10 & 0.75 & 6 & $46.15 \%$ \\
\hline 11 & 0.80 & 5 & $38.46 \%$ \\
\hline 12 & 0.85 & 4 & $30.77 \%$ \\
\hline 13 & 0.90 & 4 & $30.77 \%$ \\
\hline
\end{tabular}




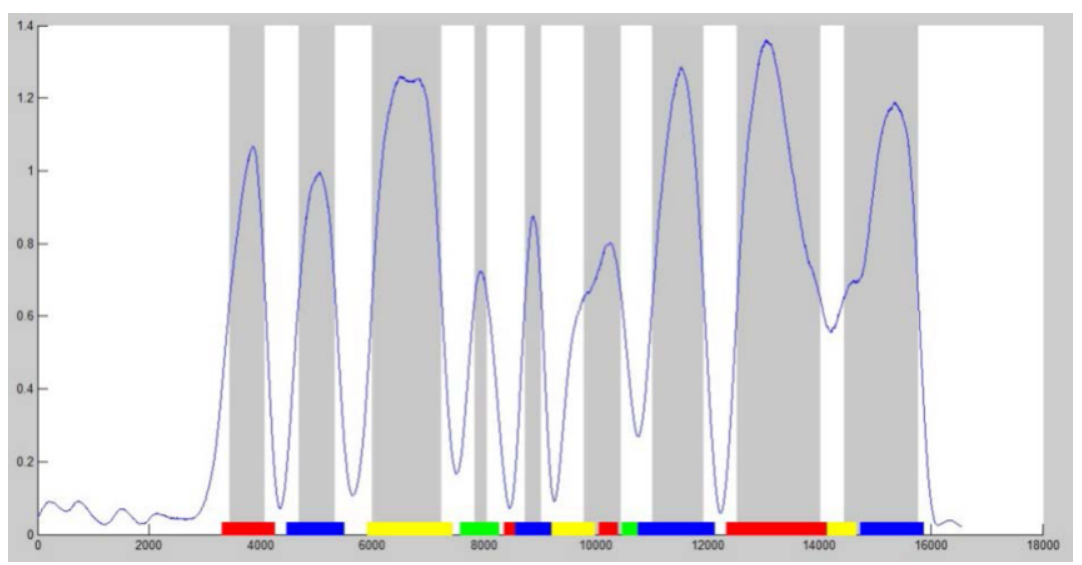

Fig. 3. Exon endpoint value and actual exon endpoint value comparison chart.

Table 5

The table of exon prediction interval

\begin{tabular}{llll}
\hline $\begin{array}{l}\text { Serial number of } \\
\text { predicted exons }\end{array}$ & $\begin{array}{l}\text { Position of predicted } \\
\text { exons }\end{array}$ & $\begin{array}{l}\text { Serial number of } \\
\text { predicted exons }\end{array}$ & $\begin{array}{l}\text { Position of predicted } \\
\text { exons }\end{array}$ \\
\hline 1 & $3445-4080$ & 6 & $9786-10459$ \\
2 & $4861-5336$ & 7 & $11020-11934$ \\
3 & $5996-7238$ & 8 & $12536-14019$ \\
4 & $7833-8056$ & 9 & $14446-14453$ \\
5 & $8734-9023$ & 10 & $14455-15777$ \\
\hline
\end{tabular}

We can find that the optimal value $c_{i}$ of human mitochondrial genome DNA sequence is 0.55 from Table 4.

(3) Fix the endpoint of exons

Exon endpoint value and actual exon endpoint value comparison chart is shown in Figure 3.

(4) Model Evaluation

By analyzing the Table 5, we can obviously see that 9 predicted exons ( the ninth is deleted because of too short) are fully consistent in addition that some error occurs on the endpoint of exons comparing with these in Table 3. In the other word, we predicted 9 exons in 13 of them, the correct rate is $70 \%$. It's better than the traditional Fourier transform method whose correct rate is $15 \%$.

\subsubsection{Gene prediction}

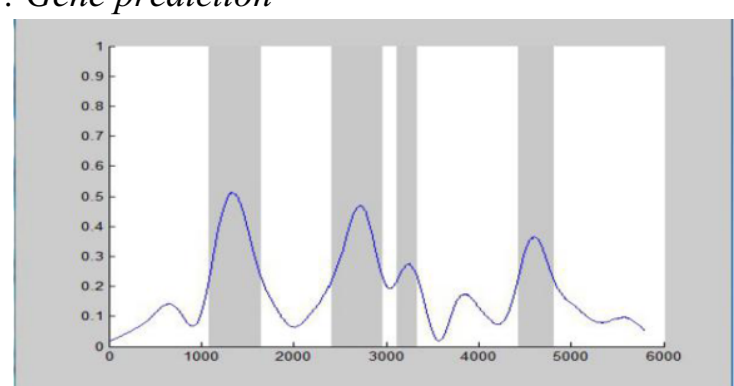

Fig. 4. Predicting outcomes of genes6-1DNA.

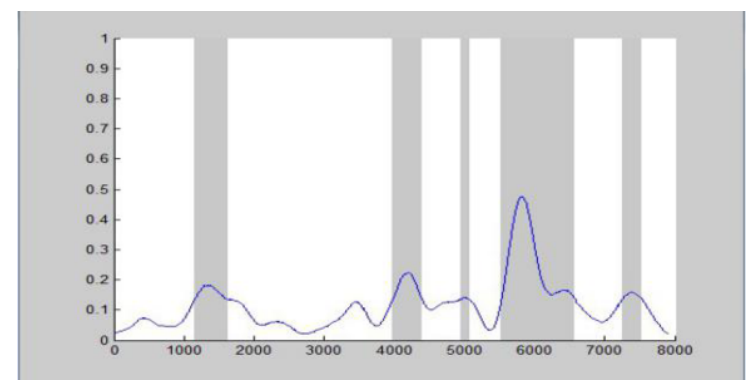

Fig. 5. Predicting outcomes of genes6-2DNA. 
Then we will predict the exons of gene6 in the title database by the improved gene identification algorithm. set $c_{i}$ as 0.7, and other value results of $c_{i}$ in annex. The results are shown in Figures 4-9 and the exon prediction interval of DNA sequence in genes6 is shown in Table 6.

\section{Conclusion}

Based on the summary of three traditional threshold determination methods, this essay put forward weighted optimization method which can show different weight and discriminant rate method of exons and introns which can increase their accuracy. We used the results of four DNA sequences to evaluate different threshold determination methods. In order to analyze DNA sequence more accurately, this paper adopted and improved short-time Fourier transform method which based on Gabor transform for gene recognition. We used traversal thoughts to transform fixed percentage of exons in the DNA sequence length to dynamic percentage which aimed at different gene types. It used the improved

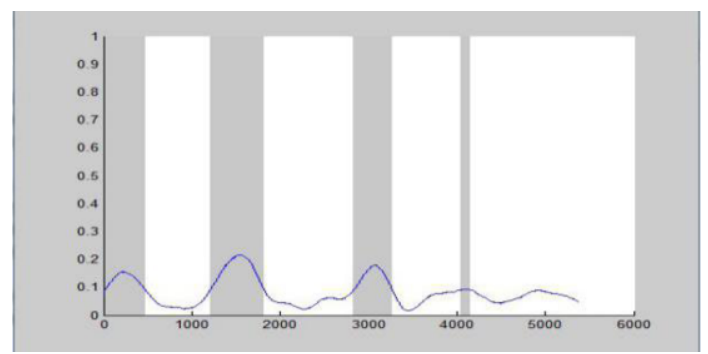

Fig. 6. Predicting outcomes of genes6-3DNA.

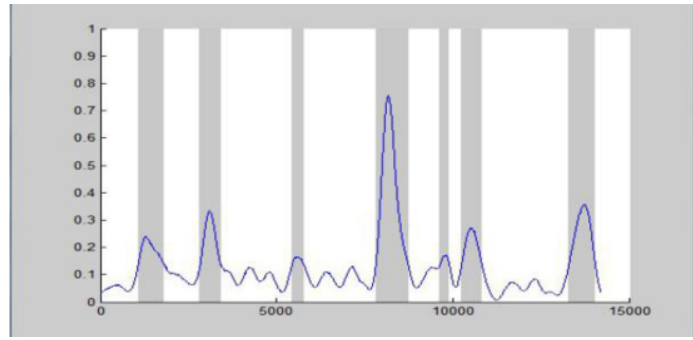

Fig. 8. Predicting outcomes of genes6-5DNA.

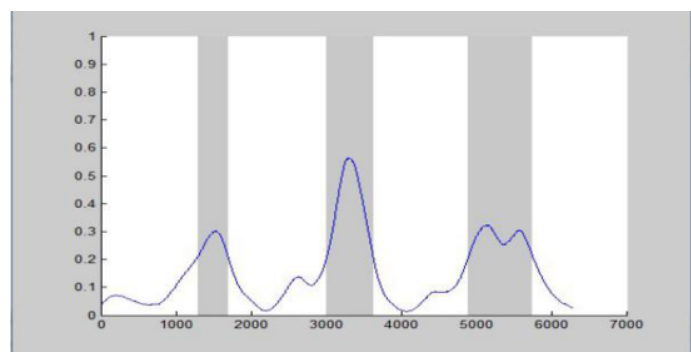

Fig. 7. Predicting outcomes of genes6-4DNA.

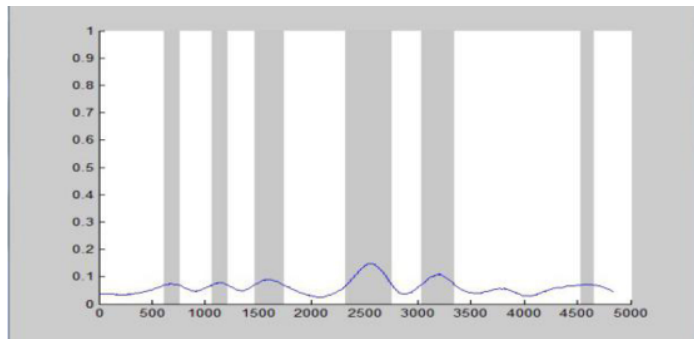

Fig. 9. Predicting outcomes of genes6-6DNA.

Table 6

Exon prediction interval of DNA sequence in genes6

\begin{tabular}{lllllll}
\hline $\begin{array}{l}\text { Serial } \\
\text { number }\end{array}$ & genes6-1 & genes6-2 & genes6-3 & genes6-4 & genes6-5 & genes6-6 \\
DNA & DNA & DNA & DNA & DNA \\
\hline 1 & $1085-1657$ & $2415-2971$ & $3123-3345$ & $4426-4821$ & - & - \\
2 & $1153-1627$ & $3978-4400$ & $4941-5070$ & $5529-6580$ & $7254-7537$ & - \\
3 & $17-474$ & $1206-1821$ & $2833-3271$ & $4049-4148$ & - & - \\
4 & $1291-1697$ & $3009-3630$ & $4987-5757$ & - & - & - \\
5 & $1073-1827$ & $2933-3427$ & $5442-5796$ & $7816-8745$ & $9631-9912$ & $10250-10834$ \\
6 & $617-762$ & $1067-1216$ & $1470-1742$ & $2327-2755$ & $3030-3351$ & $4528-4656$ \\
\hline
\end{tabular}

algorithm to identify the genes of the DNA sequence whose exons was known and compared the results with the actual endpoint of exons. It can confirm that the improved algorithm can judge out the 
endpoint of the exons more accurately. We can imagine our method can be applied to detect novel genes which RNA-seq cannot capture and is general enough to be applied to other gene identification for the other genes.

\section{Acknowledgments}

This work was financially supported by the Project of National Natural Science Foundation of China (No. 61472228), the Project of Qingdao Applied Basic Research of Qingdao (special youth project) (No. 14-2-4-55-jch), Natural Science Foundation of Shandong province (No. ZR2014FM009) and the International Cooperation Training Project of Shandong Province Outstanding Young and Middle-aged Backbone Teachers.

\section{References}

[1] S.D. Sharma, K. Shakya and S.N. Sharma, Evaluation of DNA mapping schemes for exon detection, International Conference on Computer, Communication and Electrical Technology, Tamilnadu, 2011, pp. 18-19.

[2] C. Yin and S. Yau, A Fourier characteristic of coding sequences, origins and a non-Fourier approximation, Journal of Computational Biology 9 (2005), 1153-1165.

[3] J.F. Shao, X.H. Yan and W. Shao, 3-periodicity of DNA sequence signal, Journal of Nanjing University of Technology 34 (2012), 133-137.

[4] Z. Wang, Study on gene identification using signal processing methods, Master's thesis, Tianjin University, 2010.

[5] B.S. Ma, Y.S. Zhu and Y.Z. Chen, Gene identification using multiple statistical features, Computer Engineering and Applications 45 (2009), 118-119.

[6] D. Anastassiou, Frequency-domain analysis of biomolecular sequences, Bioinformatics 16 (2000), 1073-1081.

[7] D. Kotlar and Y. Lavner, Gene prediction by spectral rotation measure: A new method for identifying protein-coding regions, Genome Research 13 (2003), 1930-1937.

[8] C. Yin and S.T. Yau, Prediction of protein coding regions by the 3-base periodicity analysis of a DNA sequence, Journal of Theoretical Biology 7 (2007), 687-694.

[9] Z.J. Liu, S.P. Feng and M.K. Pandey, Identification of expressed resistance gene analogs from peanut (arachis hypogaea L.) expressed sequence tags, Journal of Integrative Plant Biology 55 (2013), 453-461.

[10]H.B. An, H.C. Zheng and L. Zhang, Partial least squares based identification of Duchenne muscular dystrophy specific genes, Journal of Zhejiang University-SCIENCE B (Biomedicine and Biotechnology) 14 (2013), 973-982.

[11] J. Zhang, D. Xu, J. Nie, R. Han, Y. Zhai and Y. Shi, Comparative gene identification-58 (CGI-58) promotes autophagy as a putative lysophosphatidylglycerol acyltransferase, Proceedings of the National Academy of Science of the United States of America 110 (2013), 45-50.

[12] K.A. Zierler, D. Jaeger, N.M. Pollak, et al., Functional cardiac lipolysis in mice critically depends on comparative gene identification-58, The Journal of Biological Chemistry 288 (2013), 892-904.

[13] M.L. Stanly Paul, Amandeep Kaur, Ankit Geetea and M. Elizabeth Sobhia, Essential gene identification and drug target prioritization in Leishmania species, Molecular Biosystems 10 (2014), 1184-1195. 